\title{
Wigner SU(4) symmetry, clustering, and the spectrum of ${ }^{12} \mathrm{C}$
}

\author{
Shihang Shen ${ }^{1, a}$ (D), Timo A. Lähde ${ }^{1}$, Dean Lee ${ }^{2}$, Ulf-G. Meißner ${ }^{3,1,4}$ \\ ${ }^{1}$ Institut für Kernphysik, Institute for Advanced Simulation and Jülich Center for Hadron Physics, Forschungszentrum Jülich, 52425 Jülich, \\ Germany \\ ${ }^{2}$ Facility for Rare Isotope Beams and Department of Physics and Astronomy, Michigan State University, East Lansing, MI 48824, USA \\ ${ }^{3}$ Helmholtz-Institut für Strahlen- und Kernphysik and Bethe Center for Theoretical Physics, Universität Bonn, 53115 Bonn, Germany \\ 4 Tbilisi State University, 0186 Tbilisi, Georgia
}

Received: 14 June 2021 / Accepted: 6 September 2021 / Published online: 20 September 2021

(C) The Author(s) 2021

Communicated by Mark Caprio

\begin{abstract}
We present lattice calculations of the low-lying spectrum of ${ }^{12} \mathrm{C}$ using a simple nucleon-nucleon interaction that is independent of spin and isospin and therefore invariant under Wigner's SU(4) symmetry. We find strong signals for all excited states up to $\sim 15 \mathrm{MeV}$ above the ground state, and explore the structure of each state using a large variety of $\alpha$ cluster and harmonic oscillator trial states, projected onto given irreducible representations of the cubic group. We are able to verify earlier findings for the $\alpha$ clustering in the Hoyle state and the second $2^{+}$state of ${ }^{12} \mathrm{C}$. The success of these calculations to describe the full low-lying energy spectrum using spin-independent interactions suggest that either the spin-orbit interactions are somewhat weak in the ${ }^{12} \mathrm{C}$ system, or the effects of $\alpha$ clustering are diminishing their influence. This is in agreement with previous findings from ab initio shell model calculations.
\end{abstract}

\section{Motivation}

In Ref. [1], it was shown that the ground state properties of many light and medium-mass nuclei as well as neutron matter could be well-described by microscopic $A$-body lattice calculations using a spin- and isospin-independent interaction plus the Coulomb interaction. These spin- and isospinindependent interactions obey Wigner's SU(4) symmetry [2], where the four spin and isospin nucleon components transform as an SU(4) quartet. The very simple interactions in Ref. [1] have only four parameters: the strength of the twonucleon interaction, the range of the two-nucleon interaction, the range of the local part of the two-nucleon interaction, and the strength of the three-nucleon interaction. The strength and

\footnotetext{
1 The meaning of the term "local" is explained in the next section.

a e-mail: s.shen@fz-juelich.de (corresponding author)
}

range of the $\mathrm{SU}(4)$-symmetric local interaction were shown in Ref. [3] to play an important role in the effective interactions between two $\alpha$ clusters and the binding of nuclei with more than four nucleons. These findings are consistent with empirical observations seen in other calculations [4], and the underlying mechanisms of the effective cluster interactions have been studied in detail $[5,6]$.

An interesting unresolved question is whether or not such simple SU(4)-symmetric interactions can reproduce more than just the average ground state properties of atomic nuclei. It is clear that nuclei with large spin-orbit splittings among nuclear subshells will not be properly described if the nucleonic interactions are independent of spin. However, if we are considering a nuclear system where $\alpha$ clustering is important, then the impact of the spin-orbit interactions might be significantly reduced. But this also raises the possibility that some of the nuclear states are well described while others are not. Therefore, the success or failure of these simple spin-independent interactions in describing the spectrum of a given nucleus provides a useful probe for illuminating the underlying physics. We should note that several ab initio shell model calculations have found that the spin-orbit splittings are not strong for ${ }^{12} \mathrm{C}[7,8]$, suggesting that the simple $\mathrm{SU}(4)$ symmetric interactions might capture the essential physics.

In this work, we focus on the nucleus with perhaps the most interesting and astrophysically important spectrum, ${ }^{12} \mathrm{C}$. In the case of ${ }^{12} \mathrm{C}$, there is much evidence of competition between the arrangement of nucleons into shell model orbitals and the grouping of nucleons into $\alpha$ clusters [9]. For the studies here, we use a simple SU(4)-symmetric interaction that was introduced in Ref. [10] for calculations of light nuclei and light hypernuclei. In this greatly simplified interaction, there is no Coulomb interaction and no three-nucleon interaction. There are only two free parameters: the strength of the two-nucleon interaction and the range of the local part 
of the interaction. As we will show, the results using such a simple interaction are surprisingly good. While the interactions are extremely simple, we find evidence that the ${ }^{12} \mathrm{C}$ nucleus sits at an interesting tipping point where the competition between the shell structure and clustering produces a low-energy spectrum with qualitatively different types of nuclear states.

While our results here are fully microscopic $A$-body calculations with full correlations to all orders, they are not $a b$ initio in the sense that the nucleon-nucleon interaction is not fitted to nucleon-nucleon scattering data but rather tuned to reproduce the ground state energies of ${ }^{4} \mathrm{He}$ and ${ }^{12} \mathrm{C}$. Nevertheless, these calculations serve as an important comparative benchmark for future $a b$ initio calculations using chiral effective field theory.

\section{Background}

Wigner SU(4) symmetry is known to be connected to the large-color (large $N_{c}$ ) limit of QCD $[11,12]$. While the lowenergy $S$-wave nucleon-nucleon interactions should satisfy Wigner SU(4) symmetry up to effects of order $1 / N_{c}^{2}$, the existence of a bound state in the spin-triplet channel and not the spin-single channel appears to violate this result. Recently, it has been shown that the spin-isospin exchange symmetry is recovered to the expected accuracy, provided that the momentum resolution scale (or EFT cutoff) is chosen appropriately, at $\sim 500 \mathrm{MeV}$ [13], in agreement with earlier studies [14]. This also provides justification for the use of a Wigner SU(4) symmetric leading-order (LO) interaction [1] as a basis for the chiral EFT expansion.

Nuclear Lattice Effective Field Theory (NLEFT) is an $a b$ initio method where the chiral EFT expansion is combined with Projection Monte Carlo (PMC) simulations $[15,16]$. For PMC, Wigner SU(4) symmetric interactions afford significant computational advantages, due to their simplicity and minimal fermion sign oscillations. Remarkably, recent NLEFT studies have found that the interactions between $\alpha$ particles (or ${ }^{4} \mathrm{He}$ nuclei) appear fine-tuned with respect to seemingly minor details of the nucleon-nucleon interaction, in particular to the locality of the nucleon-nucleon force [3]. By a local force, we refer to the case where the positions of the particles remain unchanged by the interaction process, while a non-local force in general induces changes in the particle positions. Our first objective is to study to what extent the spectrum of ${ }^{12} \mathrm{C}$ can be described by a Wigner SU(4) symmetric two-nucleon force with local as well as non-local components. The strengths of these two interaction components are tuned to correctly reproduce the ${ }^{4} \mathrm{He}$ and ${ }^{12} \mathrm{C}$ ground-state energies.

The observed cluster phenomena in nuclear systems such as ${ }^{12} \mathrm{C}$, see e.g. [17], and the effective interactions between $\alpha$ clusters in nuclei are closely related to the locality of the Wigner SU(4) symmetric nucleon-nucleon interaction. For a review on clustering effects in light nuclei, see Ref. [9] and recent related experimental findings are given in Refs. $[18,19]$. The ground state of ${ }^{8} \mathrm{Be}$ is a quasi-bound $2 \alpha$ state formed by a short-range repulsion and a medium-range attraction of the effective $\alpha-\alpha$ interaction, which has been experimentally determined from the $\alpha \alpha$ scattering phase shifts. This $\alpha-\alpha$ interaction also describes the $3 \alpha$ structure of the "Hoyle state", i.e. the $0_{2}^{+}$state of ${ }^{12} \mathrm{C}$, as determined by previous NLEFT studies [20,21]. Our second objective is to study to what extent an (appropriately tuned) Wigner SU(4) symmetric nucleon-nucleon interaction with local and nonlocal components can describe the structure and excitation energies of the low-lying states of ${ }^{12} \mathrm{C}$, filling a gap of earlier studies that exclusively considered the even-parity states in terms of $3 \alpha$ clusters. To this end, we employ PMC simulations with $\alpha$ cluster and harmonic oscillator (HO) trial states, projected onto different irreducible representations (or irreps) of the cubic group. This allows us to draw conclusions about the spin-parity quantum numbers $J^{P}$ and the (dominant) structure of the states in question. In particular, we are able to verify the earlier conclusions from NLEFT calculations about the $\alpha$ cluster structure of the low-lying $0^{+}$and $2^{+}$states of ${ }^{12} \mathrm{C}$. As noted above, we use a simplified SU(4)symmetric interaction of the form defined in Ref. [10] with local and non-local two-nucleon interactions. The results we present here can be used as a benchmark for ab initio chiral effective field theory calculations on the lattice starting from an accurate description of nucleon-nucleon scattering [22].

This paper is organized as follows. In Sect. 3, we briefly review the interaction underlying this study. Section 4 contains the details of the calculation of the various excited states in ${ }^{12} \mathrm{C}$. In Sect. 5 we display the pertinent results for all level up to $\sim 15 \mathrm{MeV}$ above the ground state. We also discuss the interplay between $\alpha$ cluster and shell-model states. These results are discussed and put into perspective in Sect. 6.

\section{Interactions}

We have considered two choices of the spatial lattice spacing, a coarse one of $a=1.97 \mathrm{fm}$ with temporal lattice spacing $a_{t}=0.66 \mathrm{fm}$, and a finer one of $a=1.64 \mathrm{fm}$ with temporal lattice spacing $a_{t}=0.55 \mathrm{fm}$. The spatial lattice spacings correspond to momentum cut-offs of $314 \mathrm{MeV}$ and $378 \mathrm{MeV}$, respectively. All calculations use a periodic box with length $L=9$ in each spatial dimension. The physical box size for $a=1.97 \mathrm{fm}$ is then $17.7 \mathrm{fm}$, and for $a=1.64 \mathrm{fm}$ it is $14.8 \mathrm{fm}$. This is sufficiently large to suppress finite volume effects to a level that is smaller than the other sources of error in our analysis. 
Table 1 Fit results of the parameters $C_{0}$ and $s_{\mathrm{L}}$ for lattice spacings $a=1.97 \mathrm{fm}$ and $1.64 \mathrm{fm}$. The obtained ground state energies of ${ }^{4} \mathrm{He}$ and ${ }^{12} \mathrm{C}$ are also given, in comparison with experiment

\begin{tabular}{llll}
\hline & $a=1.97 \mathrm{fm}$ & $a=1.64 \mathrm{fm}$ & Exp. \\
\hline$C_{0}\left[\mathrm{MeV}^{-2}\right]$ & $-5.53 \times 10^{-6}$ & $-3.72 \times 10^{-6}$ & \\
$s_{\mathrm{L}}$ & 0.073 & 0.083 & \\
$E_{{ }{ }_{\mathrm{He}}[\mathrm{MeV}]}$ & $-28.299(9)$ & $-28.290(15)$ & -28.296 \\
$E_{12 \mathrm{C}}[\mathrm{MeV}]$ & $-92.15(3)$ & $-92.12(4)$ & -92.162 \\
\hline
\end{tabular}

We use an $N N$ interaction $V$ with Wigner SU(4) symmetry [2],

$V=\frac{C_{0}}{2} \sum_{\mathbf{n}^{\prime}, \mathbf{n}, \mathbf{n}^{\prime \prime}}: \rho_{\mathrm{NL}}\left(\mathbf{n}^{\prime}\right) f_{S_{\mathrm{L}}}\left(\mathbf{n}^{\prime}-\mathbf{n}\right) f_{S_{\mathrm{L}}}\left(\mathbf{n}-\mathbf{n}^{\prime \prime}\right) \rho_{\mathrm{NL}}\left(\mathbf{n}^{\prime \prime}\right)$,

where $C_{0}$ is a coupling constant, vector $\mathbf{n}$ labels the lattice sites, and the colons denote normal ordering. The function $f_{s_{\mathrm{L}}}$ is defined with a "local smearing" parameter $s_{\mathrm{L}}$ as

$$
\begin{cases}f_{s_{\mathrm{L}}}(\mathbf{n})=1, & |\mathbf{n}|=0, \\ s_{\mathrm{L}}, & |\mathbf{n}|=1, \\ 0, & \text { otherwise. }\end{cases}
$$

The non-local density operator $\rho_{\mathrm{NL}}(\mathbf{n})$ in Eq. (1) is

$\rho_{\mathrm{NL}}(\mathbf{n})=a_{\mathrm{NL}}^{\dagger}(\mathbf{n}) a_{\mathrm{NL}}(\mathbf{n})$,

with the non-local creation and annihilation operators defined with the "non-local smearing" parameter $s_{\mathrm{NL}}$ as

$$
\begin{aligned}
& a_{\mathrm{NL}}^{\dagger}(\mathbf{n})=a^{\dagger}(\mathbf{n})+s_{\mathrm{NL}} \sum_{\left|\mathbf{n}^{\prime}\right|=1} a^{\dagger}\left(\mathbf{n}+\mathbf{n}^{\prime}\right), \\
& a_{\mathrm{NL}}(\mathbf{n})=a(\mathbf{n})+s_{\mathrm{NL}} \sum_{\left|\mathbf{n}^{\prime}\right|=1} a\left(\mathbf{n}+\mathbf{n}^{\prime}\right) .
\end{aligned}
$$

We note that in this highly simplified nuclear interaction certain components are missing, which should be included in a more realistic calculation. While we do not consider a three-nucleon force, we find that its effects can mostly be absorbed into a renormalization of the strength of the twonucleon force. This is consistent with our finding that the ratio of $\left\langle: \rho^{3}:\right\rangle$ to $\left\langle: \rho^{2}:\right\rangle$ is approximately the same for all nuclear states of ${ }^{12} \mathrm{C}$. Similarly, the Coulomb interaction does not contribute much to the energy splitting between ${ }^{12} \mathrm{C}$ levels, and so its contribution to the binding energy can also be absorbed by a renormalization of the two-nucleon force.

Specifically, the two interaction parameters $C_{0}, s_{\mathrm{L}}$, are determined by fitting to the ground-state energies of ${ }^{4} \mathrm{He}$ and ${ }^{12} \mathrm{C}$, while $s_{\mathrm{NL}}=0.2$ is taken to equal that used in Ref. [10]. The fitted parameters and ground state energies of ${ }^{4} \mathrm{He}$ and ${ }^{12} \mathrm{C}$ are given in Table 1.

\section{Methods}

Let us first discuss the basics of our PMC simulations. We define the transfer matrix operator

$M=: \exp \left(-\alpha_{t} H\right)$,

with $\alpha_{t}=a_{t} / a$ the ratio of temporal to spatial lattice spacings. The Hamiltonian is given by

$H=T+V$,

where the interaction $V$ corresponds to Eq. (1), and the kinetic energy $T$ is taken to be

$$
\begin{aligned}
T= & \frac{3}{m_{N}} \sum_{\mathbf{n}} \rho(\mathbf{n}) \\
& -\frac{1}{2 m_{N}} \sum_{\mathbf{n}} \sum_{l=1}^{3}\left[\rho\left(\mathbf{n}, \mathbf{n}+e_{l}\right)+\rho\left(\mathbf{n}, \mathbf{n}-e_{l}\right)\right],
\end{aligned}
$$

with $e_{l}$ a unit vector along the $l$-axis, and $m_{N}=938.92 \mathrm{MeV}$ is the nucleon mass. The density operator is

$$
\begin{aligned}
\rho(\mathbf{n}) & =a^{\dagger}(\mathbf{n}) a(\mathbf{n}), \\
\rho\left(\mathbf{n}, \mathbf{n}^{\prime}\right) & =a^{\dagger}(\mathbf{n}) a\left(\mathbf{n}^{\prime}\right) .
\end{aligned}
$$

For a PMC simulation with $N_{\text {ch }}$ coupled "channels", we start from a set of initial trial states $\left|\Phi_{i}\right\rangle$ with $i=$ $1,2, \ldots, N_{\mathrm{ch}}$. One can define the Euclidean projection amplitudes at time step $N_{t}$ as

$Z_{k l}\left(N_{t}\right)=\left\langle\Phi_{k}\left|M^{N_{t}}\right| \Phi_{l}\right\rangle$.

Each of the trial states $\left|\Phi_{i}\right\rangle$ is a Slater determinant of singleparticle orbitals. These should be chosen properly, in order to get good statistics for the eigenstates of $H$ at small enough projection time, before any sign problem becomes severe.

By means of the projection amplitudes (11), we construct the "adiabatic" transfer matrix

$M_{q q^{\prime}}^{(a)}\left(N_{t}\right)=\sum_{q^{\prime \prime}} Z_{q q^{\prime \prime}}^{-1}\left(N_{t}\right) Z_{q^{\prime \prime} q^{\prime}}\left(N_{t}+1\right)$,

with eigenvalues

$\lambda_{i}\left(N_{t}\right)=\exp \left(-\alpha_{t} E_{i}\left(N_{t}\right)\right)$

such that the low-energy spectrum is given by the "transient energies"

$E_{i}\left(N_{t}\right)=-\frac{\log \left(\lambda_{i}\left(N_{t}\right)\right)}{\alpha_{t}}$,

in the limit of large projection time. To extrapolate the transient energies to $t=N_{t} a_{t} \rightarrow \infty$, the following ansatz (similar to Ref. [23]) is used:

$E_{i}(t)=\frac{E_{i}+\sum_{k=1}^{k_{\max }}\left(E_{i}+\Delta E_{k}\right) c_{i, k} e^{-\Delta E_{k} t}}{1+\sum_{k=1}^{k_{\max }} c_{i, k} e^{-\Delta E_{k} t}}$, 
where $E_{i}, \Delta E_{k}, c_{i, k}$ are fit parameters. The choice of $k_{\max }$ depends on the details of the fitting procedure.

On the lattice, the full rotational symmetry group is reduced to the finite cubic group, and the $2 J+1$ multiplet for states with angular momentum $J$ will split into subgroups of different irreducible representations (irreps). The decompositions of the first few $(J \leq 3)$ irreps with spherical harmonics $Y_{l, m}$ are [24,25]:

$$
\begin{aligned}
J=0: & A_{1}\left[Y_{0,0}\right], \\
J=1: & T_{1}\left[Y_{1,0}, Y_{1, \pm 1}\right], \\
J=2: E & {\left[\sqrt{\frac{1}{2}} Y_{2,2}+\sqrt{\frac{1}{2}} Y_{2,-2}, Y_{2,0}\right] } \\
\otimes & T_{2}\left[\sqrt{\frac{1}{2}} Y_{2,2}-\sqrt{\frac{1}{2}} Y_{2,-2}, Y_{2, \pm 1}\right] \\
J=3: & A_{2}\left[\sqrt{\frac{1}{2}} Y_{3,2}-\sqrt{\frac{1}{2}} Y_{3,-2}\right] \\
\otimes & T_{1}\left[\sqrt{\frac{5}{8}} Y_{3, \mp 3}+\sqrt{\frac{3}{8}} Y_{3, \pm 1}, Y_{3,0}\right] \\
\otimes & T_{2}\left[\sqrt{\frac{5}{8}} Y_{3, \pm 1}-\sqrt{\frac{3}{8}} Y_{3, \mp 3}, \sqrt{\frac{1}{2}} Y_{3,2}+\sqrt{\frac{1}{2}} Y_{3,-2}\right] .
\end{aligned}
$$

When the initial state $\left|\Phi_{i}\right\rangle$ does not possess a good angular momentum $J$ and projection (along $z$-axis) $J_{z}$, we project it explicitly onto a specific irrep.

Two types of initial states will be considered here. The first ones are based on $\alpha$ clusters with spatially distributed Gaussian wave packets:

$\phi(\mathbf{r})=\exp \left(-\frac{\mathbf{r}^{2}}{2 w^{2}}\right)$

with $w$ the width of the wave packet. The second ones are shell-model states with $\mathrm{HO}$ wave functions. The specific choice of each basis will be discussed in detail below. This is new compared to earlier NLEFT studies of the ${ }^{12} \mathrm{C}$ spectrum, which focused entirely on even-parity $\alpha$ cluster states. Because of this extended basis, we will be able to investigate all excited levels up to a given excitation energy (about $15 \mathrm{MeV})$.

The 3- $\alpha$ cluster states used in this work are shown in Fig. 1. These correspond to isosceles right triangles (S1), "bentarm" (obtuse triangular) configurations (S2), linear chains (S3), and acute isosceles triangles (S4). We note that linear chain configurations have also been used in DFT studies of $\alpha$ cluster states, see Ref. [26].
S1

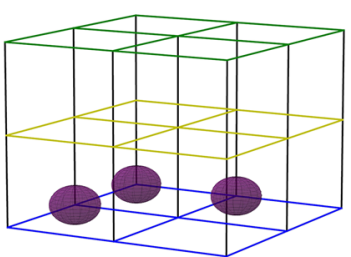

S2
S3

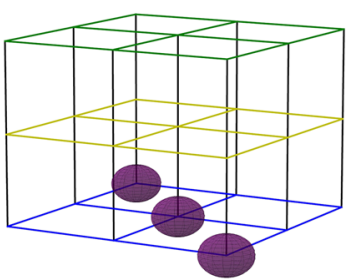

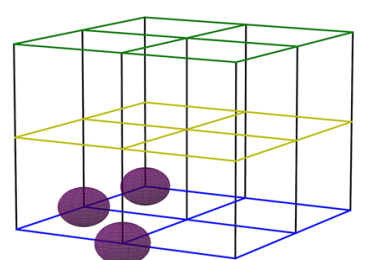

S4

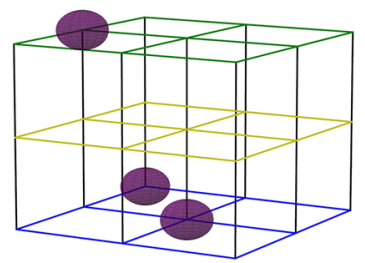

Fig. 1 Spatial configurations for 3- $\alpha$ cluster states S1, S2, S3, and S4. For S1, S2, and S3, all the three $\alpha$ clusters are on the bottom layer, while for S4 $1 \alpha$ is located on the top layer and $2 \alpha$ on the bottom layer. S1 is a isosceles right triangle, S2 is of "bent-arm" shape, S3 is a linear chain along the diagonal direction, and $\mathrm{S} 4$ is an acute isosceles triangle

\section{Results}

In this section, we show the results for various irreps that allow us to extract a number of excited states, using alphacluster and shell-model basis states [27]. In case of the shell model states, such a projection can be avoided by constructing initial states with definite $J_{z}$. This is in particular useful for the extraction of the higher spin states, as shown below.

\subsection{The irrep $A_{1}^{+}$}

First, we show the results of a two-channel PMC simulation with $A_{1}^{+}$projection (corresponding to the two lowest $0^{+}$ states), using the 3- $\alpha$ cluster configurations S1 and S2 (see Fig. 1) as initial states. The obtained transient energies versus Euclidean projection time are shown in Fig. 2. Three sets of data are obtained using wave-packet widths of $w=1.7,1.9$, and $2.1 \mathrm{fm}$, and the extrapolation fits using Eq. (15) are given by the corresponding lines. The number of exponentials in Eq. (15) in this case is chosen as $k_{\max }=3$, and will be the same for the other cases discussed in this paper unless otherwise stated. The error of the extrapolated value is indicated by the horizontal gray band.

The first $0^{+}$state (ground state) and second $0^{+}$state (Hoyle state) can be identified. As has been found in previous works [20,21], the "bent-arm" structure of S2 is suitable to search for the Hoyle state. This has been confirmed with current simulation with irrep projection of $A_{1}^{+}$. 


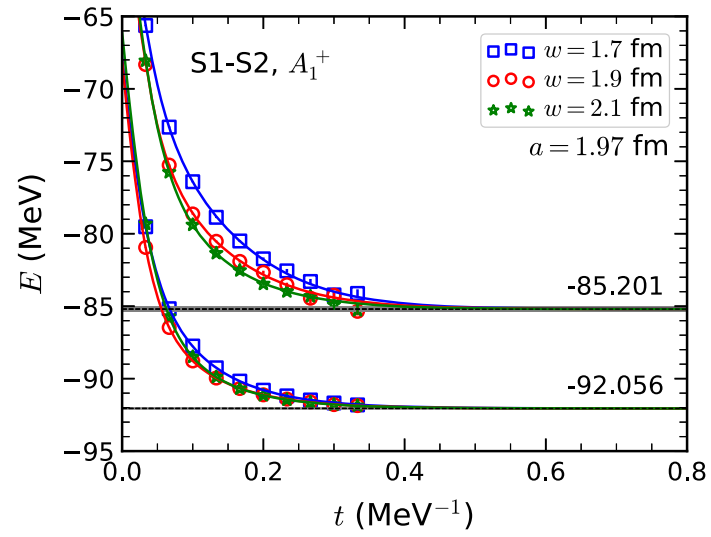

Fig. 2 Transient energies of first and second $0^{+}$state obtained by twochannel PMC with $A_{1}^{+}$projection (open symbols) versus Euclidean projection time. Three- $\alpha$ cluster configurations labelled as S1 and S2 are used as initial states and their spatial configurations are shown in Fig. 1. Three sets of data are calculated using different wave-packet width $w$, and extrapolation fits using Eq. (15) are given by corresponding lines. The error of the extrapolated value is indicated by the gray band

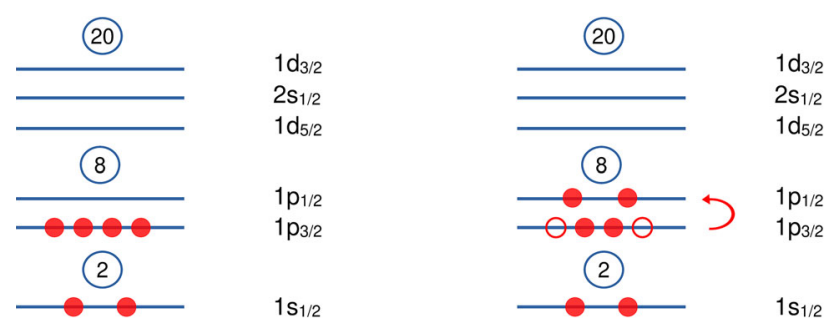

Fig. 3 Schematic drawing of ${ }^{12} \mathrm{C}$ proton shell-model trial states: (Left) ground state $|0\rangle$; (Right) $2 \mathrm{p}-2 \mathrm{~h}$ state $\left|2 p_{1 / 2} 2 p_{3 / 2}^{-1}\right\rangle$

To show the importance of the choice of initial states, we performed another two-channel PMC simulation with $A_{1}^{+}$ projection, using shell model particle-hole $\left(\left|p h^{-1}\right\rangle\right)$ states with $\mathrm{HO}$ wave functions. The configurations of protons for the two channels are shown in Fig. 3. The configurations of neutrons are always the one with the lowest energy (no particle-hole excitation) unless otherwise stated. For the first channel, the ground state $|0\rangle$ is chosen with neutrons/protons fully occupied from $1 s_{1 / 2}$ to $1 p_{3 / 2}$, while for the second channel a proton 2-particle-2-hole ( $2 \mathrm{p}-2 \mathrm{~h})$ state of $\left|2 p_{1 / 2} 2 p_{3 / 2}^{-1}\right\rangle$ with $J_{z}=0$ is used. The reason for the choice of a 2-particle2-hole is simply because this is found to give the lowest possible energy for the excited $0^{+}$. A 1-particle-1-hole $(1 \mathrm{p}-1 \mathrm{~h})$ state of $\left|p_{1 / 2} p_{3 / 2}^{-1}\right\rangle$ can only couple to $1^{+}$and $2^{+}$. The $1 \mathrm{p}-$ $1 \mathrm{~h}$ states of $\left|s_{1 / 2} s_{1 / 2}^{-1}\right\rangle$ or $\left|p_{3 / 2} p_{3 / 2}^{-1}\right\rangle$ also give an excited $0^{+}$ state, but with a much higher energy. The transient energies versus projection time are shown in Fig. 4. Three sets of data are calculated using $\mathrm{HO}$ strengths $\hbar \omega=6,10$, and $14 \mathrm{MeV}$, and extrapolation fits using Eq. (15) are given by the corresponding lines.

In Fig. 4, we also obtained a strong signal for the ground state, consistent with the one obtained in Fig. 2. However, the

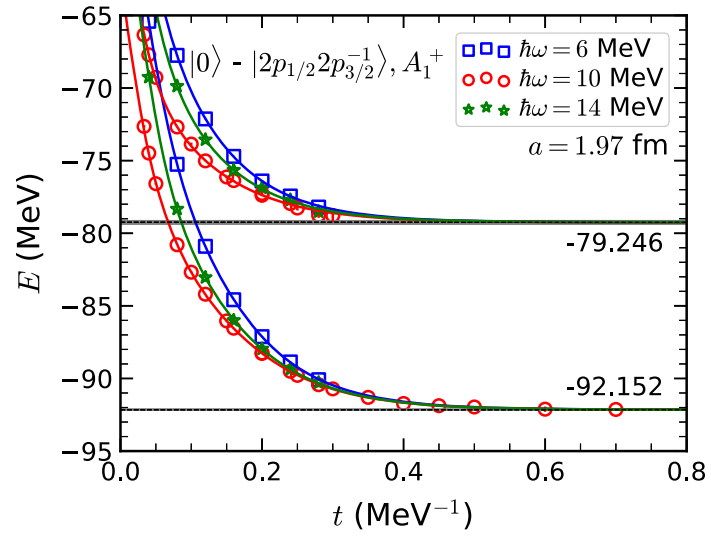

Fig. 4 Transient energies of first and third $0^{+}$states obtained by twochannel PMC with $A_{1}^{+}$projection (open symbols) versus projection time. Shell-model wave functions are used as initial states and their configurations are shown in Fig. 3. Three sets of data are calculated using HO states with different strength $\hbar \omega$

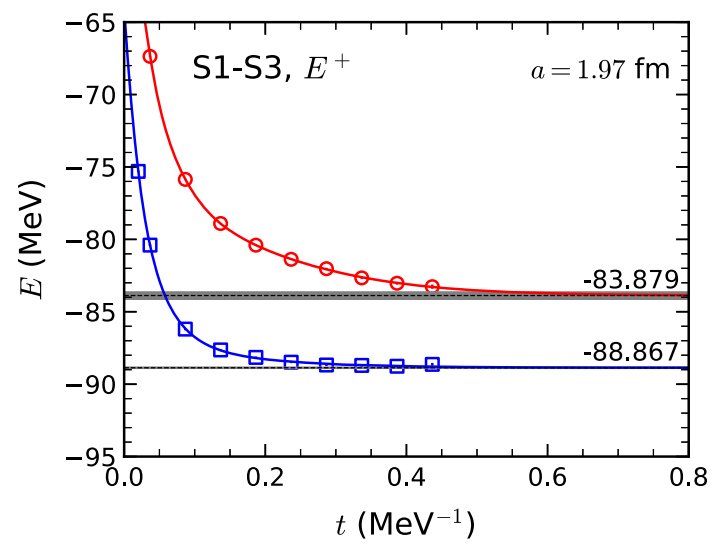

Fig. 5 Transient energies of first and second $2^{+}$state obtained by twochannel PMC with $E^{+}$projection (open symbols) versus Euclidean projection time

excited $0^{+}$state now has a much higher energy $(-79.2 \mathrm{MeV})$ than the one obtained with the bent-arm 3- $\alpha$ cluster structure $(-85.2 \mathrm{MeV})$. The second channel of the simulation with the $2 \mathrm{p}-2 \mathrm{~h}$ shell-model state does not find the Hoyle state either, but again gives us a $0^{+}$state with higher energy. This finding is consistent with observations within the no-core shell model [28-31].

\subsection{The irrep $E^{+}$}

On the one hand, while the cluster states S1 and S2 give strong signals for the first and second $0^{+}$states, the signals for the $2^{+}$states with $E^{+}$projection with the same cluster states are much less conclusive. On the other hand, the diagonal linear-chain structure $\mathrm{S} 3$ is found to work better in this case. In Fig. 5, we show the energies obtained by PMC with $E^{+}$ projection, using the $\mathrm{S} 1$ and $\mathrm{S} 3$ configurations as initial states. 


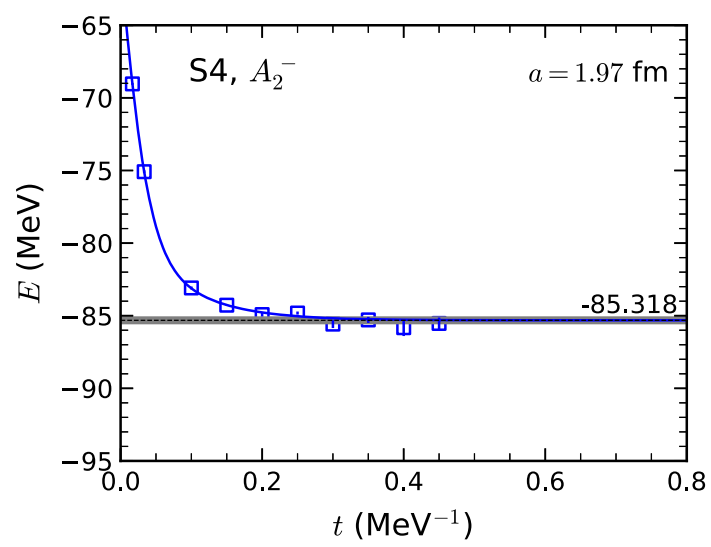

Fig. 6 Transient energies of $3^{-}$state obtained by PMC with $A_{2}^{-}$projection (open symbols) versus projection time. The spatial configuration $\mathrm{S} 4$ of the initial state is shown in Fig. 1

In Fig. 5, we can identify the first and second $2^{+}$states. Similar to the case of the $0^{+}$states, a two-channel PMC simulation using shell-model states cannot find the second $2^{+}$state, but is helpful in finding one with a higher excitation energy. As the results look similar to Fig. 4, we do not show them here. We note that our findings reinforce those of Refs. $[20,21]$, in particular concerning the notion that the second $2^{+}$state is a rotational excitation of the Hoyle state.

Using the irrep projection technique, we can search for positive parity states as well as negative parity ones, and we shall now turn to the discussion of the $3^{-}$state.

\subsection{The irrep $A_{2}^{-}$}

From Eq. (16d), one finds that $A_{2}^{-}$projection allows for a determination of the $3^{-}$state. The cluster structure $\mathrm{S} 4$ is found to be suitable in this calculation, the shape of which is shown in Fig. 1. Notably, S4 is similar to the "pear shape" of the spherical harmonics $Y_{3 \pm 2}$. The simulation results with the S4 trial state are shown in Fig. 6. As can be seen, the convergence of the energy versus projection time is fast and is achieved already around $t \simeq 0.2 \mathrm{MeV}^{-1}$. The number of exponentials in Eq. (15) in this case is $k_{\max }=2$, due to the fast convergence.

It should be noted that different projections in Eq. (16) can be used to obtain other excited states, given properly chosen initial cluster states. In some cases, shell-model states work better and irrep projection is not needed.

\subsection{Without projection}

When shell-model particle-hole wave functions are used, it is easy to construct the initial state with given angular momentum projection $J_{z}$. In the following example, we construct two states for a two-channel simulation using the $1 \mathrm{p}-1 \mathrm{~h}$ state $\left|s_{1 / 2} p_{3 / 2}^{-1}\right\rangle$, see Fig. 7, both channels having $J_{z}=1$. It is

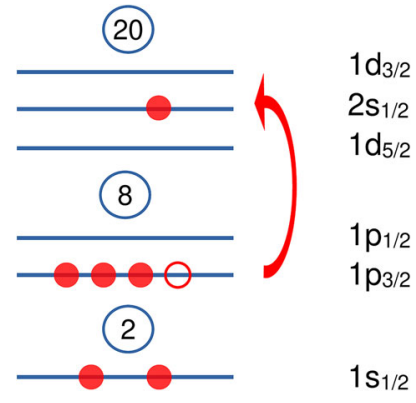

Fig. 7 Shell-model $1 \mathrm{p}-1 \mathrm{~h}$ configuration $\left|s_{1 / 2} p_{3 / 2}^{-1}\right\rangle$ of ${ }^{12} \mathrm{C}$ used to search for $1^{-}$and $2^{-}$states

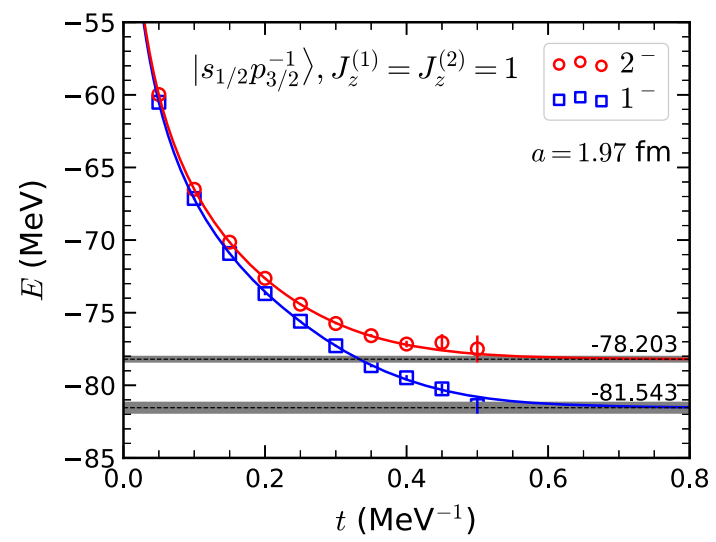

Fig. 8 Transient energies of the $1^{-}$and $2^{-}$states, obtained by twochannel PMC without irrep projection (open symbols) versus Euclidean projection time. The initial states of the two channels are $1 \mathrm{p}-1 \mathrm{~h}$ state $\left|s_{1 / 2} p_{3 / 2}^{-1}\right\rangle$, Fig. 7, both with $J_{z}=1$

straightforward to see that this choice should give us the $1^{-}$ and $2^{-}$states. With the choice of the same $J_{z}$ for both channels, the Euclidean time projection converges to the $1^{-}$and $2^{-}$states, instead of to two (degenerate) $1^{-}$or $2^{-}$states. Note that two states with both $J_{z}=0$ will work similarly. The results are shown in Fig. 8.

At small projection times, the energies in the two channels are close to each other, but the splitting eventually increases. At this point, one still cannot distinguish which one is $1^{-}$ and $2^{-}$. A separate one-channel simulation with the same configuration but $J_{z}=2$ is sufficient to uniquely identify the $2^{-}$state, and the other one will then be the $1^{-}$state. The results of the one-channel simulation with $J_{z}=2$ (not shown here) give the same energies as the red circles in Fig. 8, hence definite $J^{\pi}$ values can be assigned.

Let us summarize how some of the other excited states can be obtained in a similar way:

$-1^{+}: 1 \mathrm{p}-1 \mathrm{~h}$ state $\left|p_{1 / 2} p_{3 / 2}^{-1}\right\rangle$. Two-channel trial states are constructed, both with $J_{z}=0$ (or 1). The PMC simulation gives the $1^{+}$and $2^{+}$states. Since the $2^{+}$state has 
Table 2 Energies of the low-lying states in ${ }^{12} \mathrm{C}$ calculated by NLEFT using an SU(4) symmetric $N N$ interaction. Results for two lattice spacings are shown, in comparison with experiment [32]. Energies are given in $\mathrm{MeV}$. Errors smaller than $1 \mathrm{keV}$ are not displayed

\begin{tabular}{llll}
\hline State & $a=1.97 \mathrm{fm}$ & $a=1.64 \mathrm{fm}$ & Experiment \\
\hline $0_{1}^{+}$ & $-92.15(3)$ & $-92.12(4)$ & -92.162 \\
$2_{1}^{+}$ & $-88.87(4)$ & $-88.19(17)$ & -87.722 \\
$0_{2}^{+}$ & $-85.20(15)$ & $-85.23(22)$ & -84.508 \\
$3_{1}^{-}$ & $-84.9(2)$ & $-83.3(5)$ & $-82.521(5)$ \\
$2_{2}^{+}$ & $-83.5(2)$ & $-83.1(5)$ & $-82.29(6)$ \\
$0_{3}^{+}$ & $-80.0(3)$ & $-79.2(6)$ & $-81.9(3)$ \\
$1_{1}^{-}$ & $-81.5(4)$ & $-79.7(4)$ & $-81.315(4)$ \\
$2_{1}^{-}$ & $-78.6(2)$ & $-76.1(2)$ & $-80.326(4)$ \\
$1_{1}^{+}$ & $-79.67(11)$ & $-78.14(24)$ & $-79.452(6)$ \\
$4_{1}^{-}$ & $-78.1(2)$ & $-75.5(5)$ & $-78.846(20)$ \\
$4_{1}^{+}$ & $-80.99(11)$ & $-79.1(6)$ & $-78.083(5)$ \\
$2_{3}^{+}$ & $-79.9(4)$ & $-77.9(2)$ & -76.056 \\
$0_{4}^{+}$ & $-79.25(11)$ & $-76.94(18)$ & -74.402 \\
\hline
\end{tabular}

been identified as in Sect. 5.2, the other one will be $1^{+}$ by default.

$-4^{-}: 1 \mathrm{p}-1 \mathrm{~h}$ state $\left|d_{5 / 2} p_{3 / 2}^{-1}\right\rangle$. A one-channel trial state with $J_{z}=4$ is constructed. Since the largest $J$ value allowed by this $1 \mathrm{p}-1 \mathrm{~h}$ configuration is 4 , the PMC simulation gives the $4^{-}$state directly.

$-4^{+}: 2 \mathrm{p}-2 \mathrm{~h}$ state $\left|(\nu \pi) p_{1 / 2}(\nu \pi) p_{3 / 2}^{-1}\right\rangle$. A one-channel trial state with $J_{z}=4$ is constructed. The reason to choose $2 \mathrm{p}-2 \mathrm{~h}$ composed of 1 proton particle-hole and 1 neutron particle-hole instead of 2 protons (or neutrons) is to assure that the largest $J_{z}$ value can be 4 . When both are protons (neutrons), the largest $J_{z}$ value can only be 2 . The PMC simulation gives the $4^{+}$state.

\subsection{The ${ }^{12} \mathrm{C}$ spectrum}

To summarise the above results, the spectrum of ${ }^{12} \mathrm{C}$ below $\sim$ $15 \mathrm{MeV}$ obtained by PMC using SU(4) interaction is shown in Table 2 and Fig. 9, together with the experimental data from Ref. [32]. For $2^{+}$and $3^{-}$, the multiplet-averaged energy is used as in Ref. [25]. It is quite interesting to see that with such a simple SU(4) symmetric interaction, which was fitted to the ground states of ${ }^{4} \mathrm{He}$ and ${ }^{12} \mathrm{C}$, all the levels of ${ }^{12} \mathrm{C}$ below $15 \mathrm{MeV}$ have been found, and the agreement with experiment is surprisingly good. This suggests that our SU(4) symmetric interaction successfully incorporates many key features of the ${ }^{12} \mathrm{C}$ system.

When the lattice spacing $a=1.97 \mathrm{fm}$ is decreased to $1.64 \mathrm{fm}$, the excitation energies generally increase, with a few exceptions such as the Hoyle state. The agreement with experimental data for $a=1.64 \mathrm{fm}$ is improved for states such as $2^{+}, 3^{-}, 4^{+}$, and $0_{4}^{+}$, while for other cases the agreement appears to worsen slightly. Overall, the spectrum obtained by NLEFT is in quite good agreement with experimental data, given that only a simple SU(4) symmetric interaction has been used. Of course, the other components of the nuclear force not included here are very important and crucial to give a good universal description of the whole nuclear chart. The chiral EFT interaction up to N3LO for lattice simulation is a work in progress, and will be used in the future to investigate the spectrum of nuclei using the methods established in this work.

\section{Summary and outlook}

We have explored the low-lying spectrum of ${ }^{12} \mathrm{C}$ using a simple SU(4)-symmetric interaction with local and non-local terms. By fitting the strength of the interaction and the local smearing parameter to the ground state energies of ${ }^{4} \mathrm{He}$ and ${ }^{12} \mathrm{C}$, we have obtained a good representation of the spectrum up to excitation energies of about $15 \mathrm{MeV}$. This was achieved using initial states composed of three $\alpha$ clusters, as well as of shell model orbitals. In particular, we were able to confirm earlier NLEFT results concerning the structure of the Hoyle state and the second $2^{+}$state [21]. For the Hoyle state, prolate $\alpha$ cluster configurations are very important [33], and the second $2^{+}$state is consistent with the interpretation as a rotational excitation of the Hoyle state. Our results provide confirmation that ${ }^{12} \mathrm{C}$ sits at a fascinating balance point where the competition between the shell structure and clustering
Fig. 9 Spectrum of ${ }^{12} \mathrm{C}$ below $\sim 15 \mathrm{MeV}$ excitation energy obtained by NLEFT using an SU(4) symmetric $N N$ interaction in comparison with experiment [32]. Note that the ground state is used for tuning of the interaction

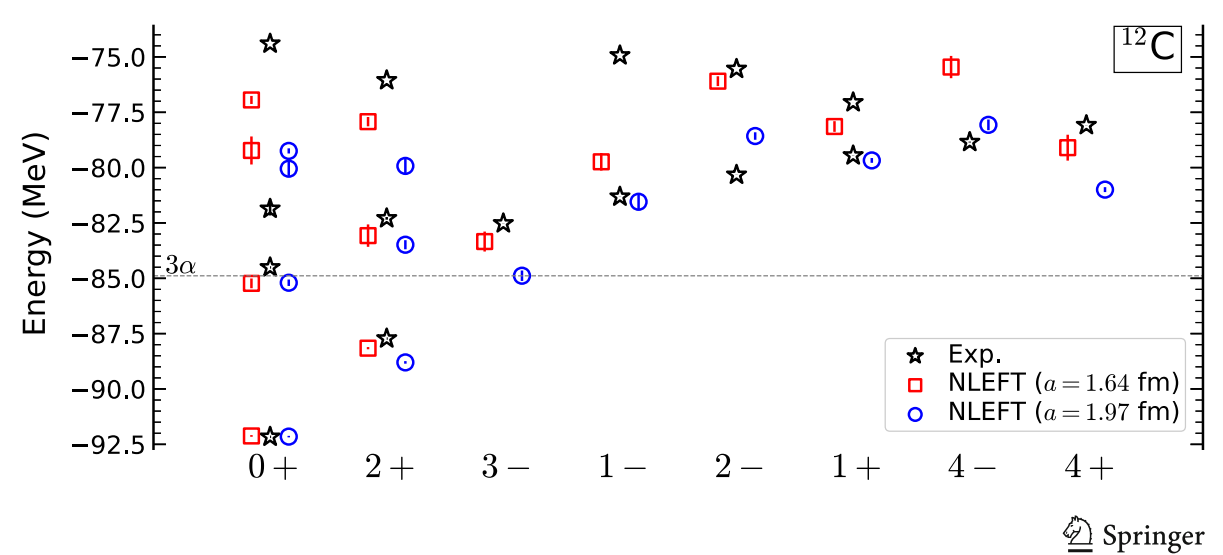


produces a low-energy spectrum with qualitatively different types of nuclear states.

The success of these simple interactions in describing all of the low-lying states of ${ }^{12} \mathrm{C}$ suggests that the tendency towards $\alpha$ clustering is probably not a simple binary attribute that effects some states of ${ }^{12} \mathrm{C}$ and not others. It is clear that the effects of $\alpha$ clustering are very prominent for the Hoyle state and the second $2^{+}$state, to the extent that their overlap with shell model initial states are so small that they cannot be detected in the lattice Monte Carlo calculations. However, it also appears that spin-orbit interactions are not playing a decisive role for the other ${ }^{12} \mathrm{C}$ states with good overlap with shell model initial states. This implies that either spinorbit interactions are somewhat weak in the ${ }^{12} \mathrm{C}$ system, or the effects of $\alpha$ clustering are diminishing their influence. This is in agreement with previous ab initio shell model calculations $[7,8]$. To clarify the underlying physics further, it would be very interesting to perform similar studies for ${ }^{16} \mathrm{O}$ and ${ }^{20} \mathrm{Ne}$ to see whether an SU(4)-symmetric interaction can fully describe the low-energy spectra of these nuclei.

Acknowledgements We thank Serdar Elhatisari, Dillon Frame, Calvin Johnson, Bing-Nan Lu and Gautam Rupak for helpful discussions. This work was supported by DFG and NSFC through funds provided to the Sino-German CRC 110 "Symmetries and the Emergence of Structure in QCD" (NSFC Grant No. 11621131001, DFG Grant No. TRR110). The work of UGM was supported in part by VolkswagenStiftung (Grant No. 93562) and by the CAS President's International Fellowship Initiative (PIFI) (Grant No. 2018DM0034). The work of DL is supported by the U.S. Department of Energy (Grant No. DE-SC0013365) and the Nuclear Computational Low-Energy Initiative (NUCLEI) SciDAC project, with computing support from the OLCF through the INCITE award "Abinitio nuclear structure and nuclear reactions". The authors gratefully acknowledge the Gauss Centre for Supercomputing e.V. (www.gausscentre.eu) for funding this project by providing computing time on the GCS Supercomputer JUWELS at Jülich Supercomputing Centre (JSC).

Funding Open Access funding enabled and organized by Projekt DEAL.

Data Availability Statement This manuscript has no associated data or the data will not be deposited. [Authors' comment: All data used in this work are freely available from the authors on request.]

Open Access This article is licensed under a Creative Commons Attribution 4.0 International License, which permits use, sharing, adaptation, distribution and reproduction in any medium or format, as long as you give appropriate credit to the original author(s) and the source, provide a link to the Creative Commons licence, and indicate if changes were made. The images or other third party material in this article are included in the article's Creative Commons licence, unless indicated otherwise in a credit line to the material. If material is not included in the article's Creative Commons licence and your intended use is not permitted by statutory regulation or exceeds the permitted use, you will need to obtain permission directly from the copyright holder. To view a copy of this licence, visit http://creativecomm ons.org/licenses/by/4.0/.

\section{References}

1. B.N. Lu, N. Li, S. Elhatisari, D. Lee, E. Epelbaum, U.-G. Meißner, Phys. Lett. B 797, 134863 (2019). [arXiv:1812.10928 [nucl-th]]

2. E. Wigner, Phys. Rev. 51, 106 (1937)

3. S. Elhatisari, N. Li, A. Rokash, J.M. Alarcón, D. Du, N. Klein, B.N. Lu, U.-G. Meißner, E. Epelbaum, H. Krebs et al., Phys. Rev. Lett. 117(13), 132501 (2016). [arXiv:1602.04539 [nucl-th]]

4. L. Contessi, A. Lovato, F. Pederiva, A. Roggero, J. Kirscher, U. van Kolck, Phys. Lett. B 772, 839-848 (2017). [arXiv:1701.06516 [nucl-th]]

5. A. Rokash, E. Epelbaum, H. Krebs, D. Lee, Phys. Rev. Lett. 118(23), 232502 (2017). [arXiv:1612.08004 [nucl-th]]

6. Y. Kanada-En'yo, D. Lee, Phys. Rev. C 103(2), 024318 (2021). [arXiv:2008.01867 [nucl-th]]

7. A.C. Hayes, P. Navratil, J.P. Vary, Phys. Rev. Lett. 91, 012502 (2003). [arXiv:nucl-th/0305072 [nucl-th]]

8. C.W. Johnson, Phys. Rev. C 91(3), 034313 (2015). [arXiv:1409.7355 [nucl-th]]

9. M. Freer, H. Horiuchi, Y. Kanada-En’yo, D. Lee, U.-G. Meißner, Rev. Mod. Phys. 90(3), 035004 (2018)

10. D. Frame, T.A. Lähde, D. Lee, U.-G. Meißner, Eur. Phys. J. A 56(10), 248 (2020). [arXiv:2007.06335 [nucl-th]]

11. D.B. Kaplan, M.J. Savage, Phys. Lett. B 365, 244-251 (1996). [arXiv:hep-ph/9509371 [hep-ph]]

12. D.B. Kaplan, A.V. Manohar, Phys. Rev. C 56, 76-83 (1997). [arXiv:nucl-th/9612021 [nucl-th]]

13. D. Lee, S. Bogner, B. A. Brown, S. Elhatisari, E. Epelbaum, H. Hergert, M. Hjorth-Jensen, H. Krebs, N. Li and B. N. Lu, et al. [arXiv:2010.09420 [nucl-th]]

14. V.S. Timoteo, S. Szpigel, E. Ruiz Arriola, Phys. Rev. C 86, 034002 (2012). [arXiv:1108.1162 [nucl-th]]

15. D. Lee, Prog. Part. Nucl. Phys. 63, 117 (2009). [arXiv:0804.3501 [nucl-th]]

16. T.A. Lähde, U.-G. Meißner, Lect. Notes Phys. 957, 1 (2019)

17. M. Gai, R. Bijker, M. Freer, T. Kokalova, D.J. Marin-Lambarri, C. Wheldon, J. Phys: Conf. Ser. 569(1), 012011 (2014)

18. R. Smith, M. Gai, M.W. Ahmed, M. Freer, H.O.U. Fynbo, D. Schweitzer, S.R. Stern, Phys. Rev. C 101(2), 021302 (2020). [arXiv:2001.07223 [nucl-ex]]

19. K. C. W. Li, F. D. Smit, P. Adsley, R. Neveling, P. Papka, E. Nikolskii, J. W. Brümmer, L. M. Donaldson, M. Freer and M. N. Harakeh, et al. [arXiv:2011.10112 [nucl-ex]]

20. E. Epelbaum, H. Krebs, D. Lee, U.-G. Meißner, Phys. Rev. Lett. 106, 192501 (2011). [arXiv:1101.2547 [nucl-th]]

21. E. Epelbaum, H. Krebs, T.A. Lähde, D. Lee, U.-G. Meißner, Phys. Rev. Lett. 109, 252501 (2012). [arXiv:1208.1328 [nucl-th]]

22. N. Li, S. Elhatisari, E. Epelbaum, D. Lee, B.N. Lu, U.-G. Meißner, Phys. Rev. C 98(4), 044002 (2018). [arXiv:1806.07994 [nucl-th]]

23. T.A. Lähde, E. Epelbaum, H. Krebs, D. Lee, U.-G. Meißner, G. Rupak, J. Phys. G 42(3), 034012 (2015). [arXiv:1409.7538 [nuclth]]

24. R.C. Johnson, Phys. Lett. B 114, 147-151 (1982)

25. B.N. Lu, T.A. Lähde, D. Lee, U.-G. Meißner, Phys. Rev. D 90(3), 034507 (2014). [arXiv:1403.8056 [nucl-th]]

26. Z.X. Ren, P.W. Zhao, J. Meng, Phys. Lett. B 801, 135194 (2020). [arXiv:2001.02834 [nucl-th]]

27. B. N. Lu, private communication (2021)

28. P. Navrátil, J.P. Vary, B.R. Barrett, Phys. Rev. Lett. 84, 5728-5731 (2000). [arXiv:nucl-th/0004058 [nucl-th]] 
29. P. Navrátil, V.G. Gueorguiev, J.P. Vary, W.E. Ormand, A. Nogga, Phys. Rev. Lett. 99, 042501 (2007). [arXiv:nucl-th/0701038 [nuclth]]

30. R. Roth, J. Langhammer, A. Calci, S. Binder, P. Navrátil, Phys. Rev. Lett. 107, 072501 (2011). [arXiv:1105.3173 [nucl-th]]

31. B.R. Barrett, P. Navrátil, J.P. Vary, Prog. Part. Nucl. Phys. 69, 131$181(2013)$
32. J.H. Kelley, J.E. Purcell, C.G. Sheu, Nucl. Phys. A 968, 71-253 (2017)

33. A.C. Dreyfuss, K.D. Launey, T. Dytrych, J.P. Draayer, R.B. Baker, C.M. Deibel, C. Bahri, Phys. Rev. C 95(4), 044312 (2017). [arXiv:1611.00060 [nucl-th]] 